inflammation, for example, alcohol and the toxins of tubercle, and syphilis. Cholelithiasis is the most important and frequent of all these causes. Associated with gall.stone disease all stages of chronic pancreatitis are met with, but most commonly the head of the pancreas is the part most affected. In such case the head of the gland will be found thickened and harder than normal. The treatment of chronic pancreatitis consists of the performance of a cholecystostomy with prolonged drainage of the gall bladder and bile ducts.

$$
1 \text { Practitioner, Aug., } 1903 .
$$

\section{INTRA-UTERINE AMPUTATIONS.}

In spite of the attention which has been given of recent years to the diseases and deformities of the fotus, it is somewhat curious that so much uncertainty exists regarding the circumstances which every now and then cause a child to be born minus one or more digits, or the distal part of a limb. It is generally allowed that something of the nature of an amputation has taken place, and indeed the appearance of the stump usually suggests this, but the mechanism by which the amputation is effected is largely a matter of conjecture. Allied to the cases in which there is an actual loss of a member are others in which a groove or constriction is cut more or less deeply into the soft tissues of a limb. It is reasonable to suppose that these are instances of incomplete amputation, and this suggestion is supported by the fact that occasionally, with loss of one member, such a constriction is found on one or other of the remaining limbs. Hence it is in the highest degree probable that both amputations and constrictions result from the action of corresponding agents. The theory that these agents are extra-fœtal bands, arising perhaps from inflammatory adhesions between the cutis and the amnion in an early stage of development, is sometimes opposed on the ground that such amputations occur in cases where no evidence of the existence of these adhesions can be detected. But it must be remembered that these formations, if they occur, are produced at a very early stage, and that there is therefore abundant opportunity for their removal by absorption. The same consideration helps to explain a failure to find the separated digit or limb. Further, there is positive evidence in the occasional discovery of partially separated digits actually surrounded or grasped by bands of lowly organised tissue. A further question which arises for discussion is whether the umbilical cord can possibly act as a constricting or amputating agent. It is certain that the cord is sometimes found in constrictions round the limbs. This is so far prima facie evidence. But it is objected that the cord is too soft a structure to cut through the tissues, and that it may readily slip into grooves formed by some other agency. It must, however, be remembered that in early development the limbs cannot offer anything like firm resistance, and that a continuous strangulation even by a soft ligature may be sufficient to compress the blood-vessels and even to divide the tissues. The balance of evidence seems decidedly to support the view that extra-fœetal bands, including the umbilical cord, are responsible for the occurrence of intra-uterine amputations and constrictions.

\section{THE CURE OE HERNIA.}

IN an address delivered recently before the SouthWest London Medical Society, at the Bolingbroke Hospital, Sir Victor Horsley discussed the present methods of dealing with inguinal and femoral herniæ. The troubles which a rupture gave rise to, he said, might be classed under the two heads of discomfort and danger. The former of these was amply sufficient to justify operative methods. He contended that to apply a truss was not to treat the hernia, as it was only in the case of congenital hernia that a cure would be effected by this means. On the other hand a surgical operation offered such a good chance of permanent relief that it was called for in nearly every case ; that, in fact, it was the only efficient and correct treatment unless some special contra-indication was present such as old age or debility. The dangers of surgical interference consisted of the risks of the anæsthesia and the risks of sepsis. Both of these he considered to be negligible in practice, and he thought it was justifiable in the case of a nervous subject to say that there was no danger at all. No fatality had occurred among the large number of such operations performed by his colleagues and himself during the last five years at University College Hospital. Sir Victor Horsley referred to the great difficulties that were in the way of ascertaining the ultimate results of surgical operations, and he thought it would be an excellent thing if an association were started with the object of finding out and recording after results. He was of opinion himself that there would be no recurrence of hernia if the operation were performed aright, but that faulty technique was responsible for a recrudescence of the trouble when such did occur. In inguinal hernia the operation which was most likely to lead to a permanent cure included a displacement of the lowest portion of the obliquus internus so as to fill up the weak spot in the abdominal wall with a layer of muscular tissue, as this was not liable to stretch and give way under pressure from the abdominal viscera. If there were an undescended testicle associated with the hernia, Sir Victor Horsley did not think it was right treatment to remove the testicle. He had yet to meet the case where it was impossible to place the testicle in the scrotum. With regard to femoral hernia he pointed out that that the femoral canal in an adult is from 1 to $1 \frac{1}{4}$ inches long, and in a short but very interesting historical review of the various operations which have been from time to time devised for the cure of the rupture he demonstrated that the earliest procedures were limited to closing the lower end of the canal and that subsequently there had been a constant tendency with each new operation invented to attack a still higher portion of the canal with a view to its occlusion, but so far no procedure had been introduced which satisfactorily closed the very mouth of the canal. He went on to describe an operation which he had devised for satisfactorily attaining this end. The method was as follows :-An incision was made above and parallel to Poupart's ligament. The obliquus externus was divided along this incision, and then without further cutting, by retracting upwards the arching fibres of the transversalis and internal oblique the upper opening of the femoral canal was brought into view. The hernial sac was then emptied, its neck ligatured, and the sac was 